\title{
A proteomic analysis of liver after ethanol binge in chronically ethanol treated rats
}

\author{
Annayya R Aroor ${ }^{1}$, Lowery J Roy², Ricardo J Restrepo ${ }^{1}$, Brian P Mooney ${ }^{2,3}$ and Shivendra D Shukla ${ }^{1 *}$
}

\begin{abstract}
Background: Binge ethanol in rats after chronic ethanol exposure augments necrosis and steatosis in the liver. In this study, two-dimensional gel electrophoresis proteomic profiles of liver of control, chronic ethanol, control-binge, and chronic ethanol- binge were compared.

Results: The proteomic analysis identified changes in protein abundance among the groups. The levels of carbonic anhydrase 3 (CA3) were decreased after chronic ethanol and decreased further after chronic ethanol-binge. Ethanol binge alone in control rats had no effect on this protein suggesting its possible role in increased susceptibility to injury by binge after chonic ethanol treatment. A protein spot, in which both cytosolic isocitrate dehydrogenase $(\mathrm{IDH} 1)$ and glutamine synthetase (GS) were identified, showed a small decrease after chronic ethanol binge but western blot demonstrated significant decrease only for glutamine synthetase in chronic ethanol treated rats. The level of gluathione S-transferase mu isoform (GSTM1) increased after chronic ethanol but was lower after chronic ethanol-binge compared to chronic ethanol treatment. The protein levels of the basic form of protein disulfide isomerase associated protein 3 (PDIA3) were significantly decreased and the acidic forms were increased after chronic ethanol- binge but not in chronic ethanol treated rats or ethanol binge in control rats. The significant changes in proteome profile in chronic ethanol binge were accompanied by a marked increase in liver injury as evidenced by enhanced steatosis, necrosis, increased 4-hydroxynonenal labeled proteins, CYP2E1 expression, and decreased histone H2AX phosphorylation.

Conclusions: Given the role of CA3, IDH1 and GST in oxidative stress; PDIA3 in protein quality control, apoptosis and DNA repair and decreased glutamine synthetase as a sensitive marker of pericentral liver injury this proteome study of chronic ethanol-binge rat model identifies these proteins for the first time as molecular targets with potential role in progression of liver injury by binge ethanol drinking.
\end{abstract}

Keywords: Binge ethanol, Chronic ethanol, Liver proteomics

\section{Background}

Alcoholic liver disease is a worldwide health problem [1,2]. The mechanisms that cause progression of liver injury are complex. Healthy liver is resistant to the action of ethanol and most individuals consuming alcohol have steatosis but not steatohepatitis [3]. The progression of steatosis to steatohepatitis has been shown to be dependent on additional factors such as endotoxin, nutritional factors, and other disease states such as hepatitis $C$ viral infection [4-7]. In this regard, binge drinking habit in chronic alcoholics is one of the most important factors contributing to the

\footnotetext{
* Correspondence: shuklasd@missouri.edu

'Department of Medical Pharmacology \& Physiology, University of Missouri,

Columbia, MO 65212, USA

Full list of author information is available at the end of the article
}

progression of alcoholic liver injury [8-11]. We have recently developed a chronic ethanol-binge rat model in which short term chronic ethanol treatment for 4 weeks does not result in significant liver injury. When these rats were subjected to 3 episodes of repeat ethanol binge it dramatically amplified liver injury. This rat model mimics findings similar to humans particularly during early alcoholic liver injury [12]. Therefore, it offers an opportunity to determine the effects of binge after chronic ethanol intake and to explore the underlying mechanisms of enhancement of liver injury by binge ethanol.

Recent developments of systems biology approaches including genomics, metabolomics and proteomics offer a better insight into the mechanisms of cellular injury and identification of protein targets without a prior

\section{Biomed Central}


knowledge of the specific underlying molecular pathway [13]. Although gene expression has provided valuable information on transcriptional regulation, significant discrepancy occurs between changes in RNA and protein expression since not all expressed genes are translated into protein products [14]. Two dimensional electrophoresis in combination with mass spectrometry (2DEMS), has been widely applied for the analyses of protein expression and their post translational modifications [15]. Proteome studies on liver have been useful in elucidating the biomarkers of liver damage and factors that contribute to susceptibility of liver tissue to drug or disease induced liver injury [14-16]. Although, liver proteomic studies have been reported for chronic ethanol treated rats [17-22], proteomics after ethanol binge drinking has not been examined. We have therefore undertaken a 2-dimensional (2D) gel-based proteomics study of liver subjected to binge ethanol administration in vivo.

\section{Results}

Chronic ethanol, control-binge and chronic ethanol-binge display differential expression of proteins

Rats were divided into four groups: control (pair-fed isocaloric diet), chronic ethanol (4 weeks), control-binge (pairfed followed by 3 ethanol binge), and chronic ethanolbinge (chronic ethanol 4 weeks followed by 3 binge). Three samples from each group were included for proteome analysis. Figure 1 shows representative 2D gel maps of liver proteins obtained from four groups. A total of 600-725 spots were resolved on these Coomassie Brilliant Blue (CBB) stained 2D gels. Spot volumes (area $\mathrm{x}$ intensity) were determined as a measure of protein expression. Spots that showed 1.5 fold increase or decrease were included in the analysis and proteins that are present in appreciable amounts were picked for identification by mass spectrometry (Figure 1).

Eight proteins that were affected by either chronic ethanol or chronic-ethanol binge were selected for
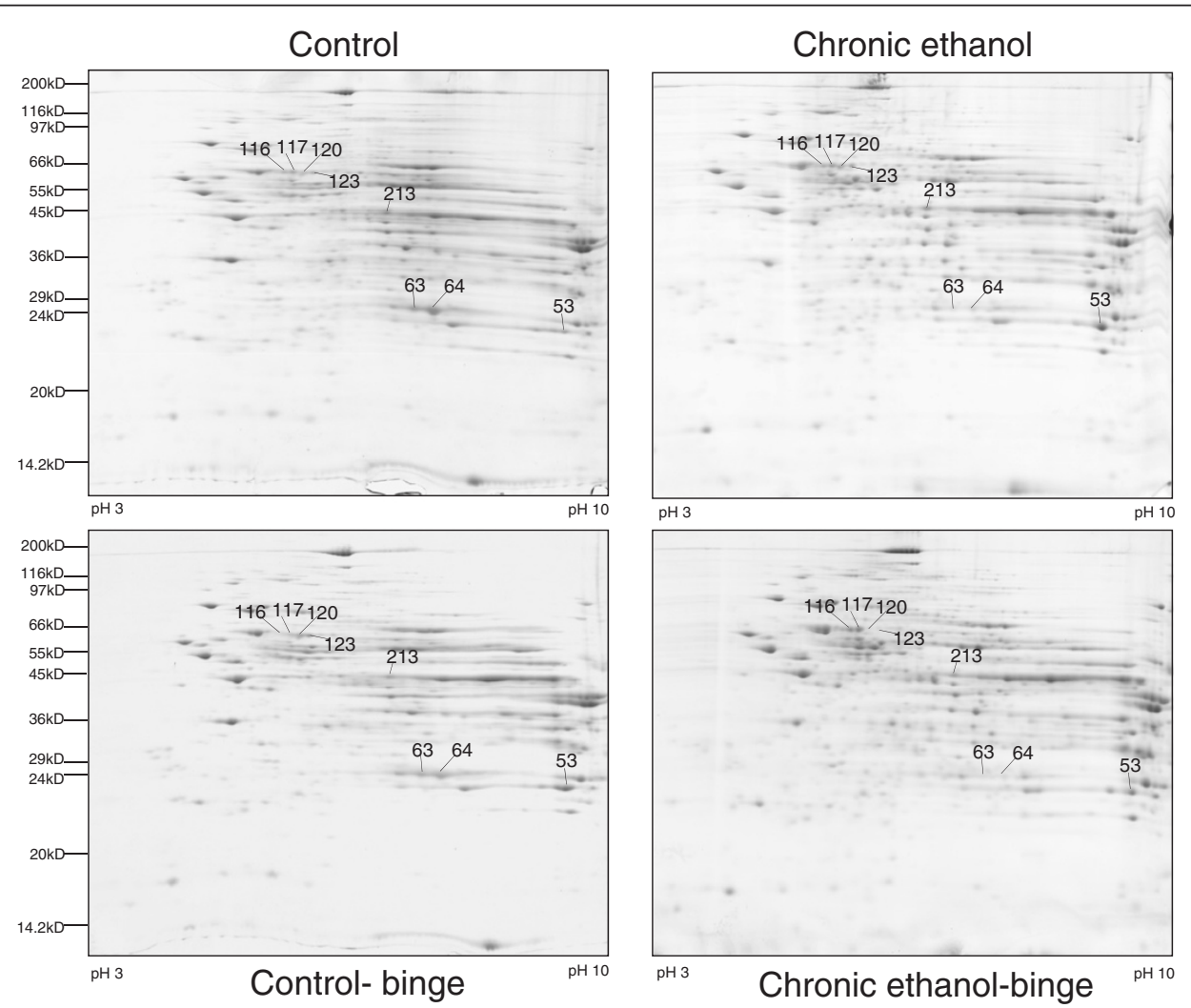

Figure 1 Composite images of 2D gels of proteins extracted from liver of control, chronic ethanol, control-binge, and chronic ethanolbinge treated rats. Rats were fed ethanol in liquid diet chronically for 4 weeks and then given three ethanol binges (5 g/kg) at 12 hr intervals. Four hours after the last dose liver samples were frozen in liquid nitrogen and stored for proteome studies. Proteins were extracted by phenol and methanol/ammonium acetate precipitation and 2D electrophoresis and analysis were performed as described under Materials and Methods. Figure 1 represents a composite image of the gel and the proteins that are differentially expressed between the treatment groups and the protein sequenced are shown by spot numbers. Control represents pair -fed animals that were given water for binge control. $\mathrm{N}=3$ rats for each group. 
identification. These proteins were excised from the gel, digested with trypsin, and subjected initially to MALDITOF/TOF MS + MS/MS analysis. Protein identification by MALDI-TOF/TOF was subsequently confirmed by peptide sequences obtained by acquiring multiple LC/ MS/MS spectra for each sample. The results are shown in Table 1. Spots 63 \& 64 were identified as carbonic anhydrase 3 (CA3) and spots 116, 117, 120 \& 123 were identified as isoforms of protein disulfide isomerase associated protein 3 (PDIA3). Spot 213 contained two proteins identified as cytosolic isocitrate dehydrogenase (IDH1) and glutamine synthetase (GS). Spot 53 was identified as glutathione $\mathrm{S}$ transferase, $\mathrm{mu}$ form (GSTM1).

The levels of CA3 decreased after chronic ethanol (Figure 2). Its levels decreased further after chronic ethanol followed by binge. In the control-binge group, CA3 levels were not significantly changed suggesting its role in increased injury by binge ethanol in chronic ethanol treated rats. We have also determined protein levels of CA3 by western immunoblot (Figure 2). The results were in agreement with the proteomic analysis. The spot volume representing combined protein levels of cytosolic isocitrate dehydrogenase/glutamine synthetase in

Table 1 Identification of differentially expressed protein spots by mass spectrometry

\begin{tabular}{|c|c|c|c|c|c|}
\hline Spot No. & Identification $^{a}$ & $\begin{array}{c}\text { Accession } \\
\text { No. }\end{array}$ & $\begin{array}{l}\mathrm{MW} \\
(\mathrm{Da})^{\mathrm{b}}\end{array}$ & $\begin{array}{l}\text { Total Ion } \\
\text { Score }\end{array}$ & $\begin{array}{c}\text { No. } \\
\text { peptides } \\
\text { matched }\end{array}$ \\
\hline 63 & $\begin{array}{l}\text { Carbonic } \\
\text { anhydrase } 3\end{array}$ & 3137484 & 29698 & 206 & 7 \\
\hline 64 & $\begin{array}{l}\text { Carbonic } \\
\text { anhydrase } 3\end{array}$ & 3137484 & 29698 & 505 & 12 \\
\hline 213 & $\begin{array}{l}\text { Cytosolic isocitrate } \\
\text { dehydrogenase }^{d}\end{array}$ & 6647554 & 47030 & 202 & 10 \\
\hline 213 & $\begin{array}{l}\text { Glutamine } \\
\text { synthetase }^{d}\end{array}$ & 204349 & 43001 & 133 & 5 \\
\hline 53 & $\begin{array}{l}\text { Glutathione S } \\
\text { transferase M1 }\end{array}$ & 204503 & 26127 & 674 & 17 \\
\hline 116 & $\begin{array}{l}\text { Protein disulfide } \\
\text { isomerase } \mathrm{A} 3\end{array}$ & 1352384 & 57044 & 163 & 6 \\
\hline 117 & $\begin{array}{l}\text { Protein disulfide } \\
\text { isomerase A3 }\end{array}$ & 1352384 & 57044 & 797 & 22 \\
\hline 120 & $\begin{array}{l}\text { Protein disulfide } \\
\text { isomerase A3 }\end{array}$ & 1352384 & 57044 & 994 & 28 \\
\hline 123 & $\begin{array}{l}\text { Protein disulfide } \\
\text { isomerase } A 3\end{array}$ & 1352384 & 57044 & 1294 & 30 \\
\hline
\end{tabular}

${ }^{\mathrm{a}}$ Data from LCMS runs were searched against NCBInr limited to mammals using MASCOT V2.2.

${ }^{\mathrm{b}}$ This is the predicted molecular weight based on the translated gene sequence.

'The total ion score from the MASCOT report. Individual peptide ion scores $>36$ have significant identify or homology $(p<0.05)$.

${ }^{\mathrm{d}}$ Two proteins were confidently identified in spot number 213 . Based on number of peptides matched, isocitrate dehydrogenase is likely the more abundant of the two. proteome analysis was decreased by about $40 \%$ after chronic ethanol-binge (Figure 3). However, the western blot results showed only a small decrease in the protein levels of cytosolic IDH1 in all the treated groups. Interestingly, protein levels of glutamine synthetase decreased in the chronic ethanol-binge group (Figure 3). In the proteome analysis, GSTM1 levels were increased after chronic ethanol and control binge but the levels are lower in chronic ethanol-binge compared to chronic ethanol (Figure 4). However, western immunoblot did not reveal significant increase after chronic ethanol (Figure 4). PDIA3 was identified in multiple forms with shifting of isoelectric point (pI). The protein levels of the more basic forms (spots 120 and 123) were lower in the chronic ethanol binge group whereas the protein levels of the more acidic forms (spots 116 and 117) were higher in the chronic ethanol binge group (Figure 5). The western immunoblot did not reveal any significant change in the expression of PDIA3 in any of the groups (Figure 5). We therefore calculated the sum of the density of all the forms in proteome analysis and observed that the changes in all the three treated groups were not different from the control group (data not shown). These results suggest that, changes in the levels of different isoforms seen after chronic-ethanol binge may represent post translational modifications of the protein. The shift in charge may be due to phosphorylation of PDIA3 forms since phosphorylation of PDIA3 has been shown to occur in vivo and such alterations are associated with a decrease in protein folding function of PDIA3 [23]. To examine this possibility, we performed pro-Q staining that detects phosphorylated forms. Pro Q stain showed significant decrease and increase in several phosphorylated proteins (Figure 6). However, the phosphorylation status of those proteins that showed differential changes in Coomassie blue staining (Table 1) were not altered. It may be mentioned that the quantitative analysis of these phosphorylated spots showed a trend for lowered phosphorylation for basic forms of PDIA3 and increased phosphorylation for acidic form (data not shown).

\section{Relationship of proteome changes to liver injury during chronic ethanol and chronic ethanol-binge}

CA3 and IDH1 have been shown to exert protection against oxidative stress and liver injury [24,25]. We examined hepatic steatosis in different groups. The results are shown in Figure 7 A-D. Steatosis was moderate in the chronic ethanol group or control ethanol binge. In contrast, stetaosis was more marked in chronic ethanol binge group. Another parameter of hepatotoxic effects of alcohol is increased expression of CYP2E1 [26,27]. Compared to control, a moderate increase (1.5 to 2 fold) in CYP2E1 protein expression was noted after chronic ethanol and 


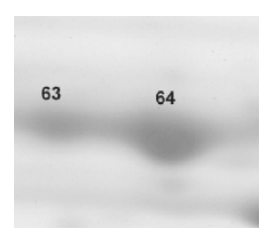

Control

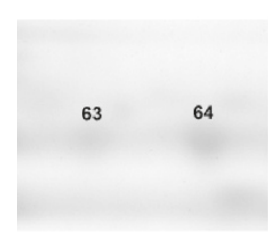

Chronic ethanol

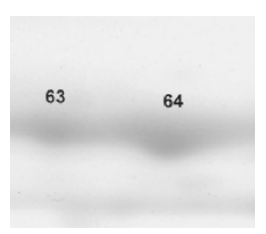

Control- binge

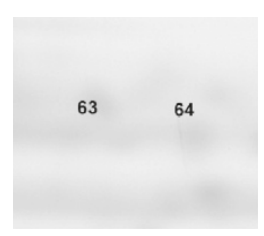

Chronic ethanolbinge

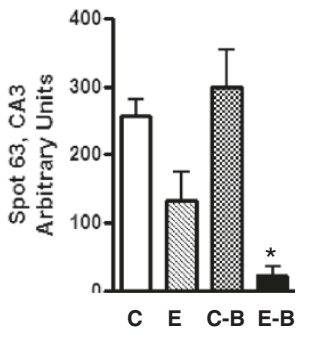

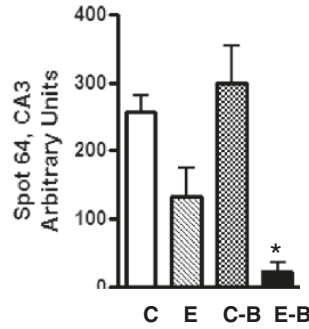

C

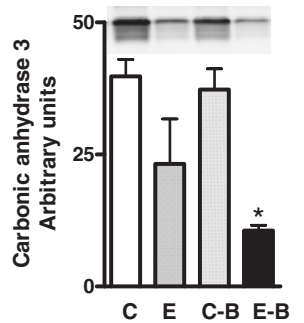

Western analysis

Figure 2 Quantitative analysis of carbonic anhydrase 3 isoforms from 2D gels and western immunoblot analysis. Rats were fed ethanol in liquid diet chronically for 4 weeks and then given 3 binge $(5 \mathrm{gm} / \mathrm{kg})$ at $12 \mathrm{hr}$ intervals and samples were collected as in Figure 1 . The levels of carbonic anhydrase 3 were determined by western immunoblot as described under Materials and Methods. Control represents pair -fed animals that were given water for binge control. Values are mean $\pm \mathrm{SE}(\mathrm{n}=3$ rats for each group). Western blot represents a typical experiment.

${ }^{*}$ significant from control group ( $p<0.05$ ); C: Control (pair fed); E: Chronic ethanol; C-B: Control- ethanol binge; E-B: Chronic-ethanol binge.

acute three binge whereas a marked increase (about 4 fold) in CYP2E1 was seen after chronic ethanol-binge (Figure 7E). We have also determined the pattern of protein oxidation using antibodies against 4-hydroxynonenal
(4-HNE). The increase in 4-HNE is not only an index of cellular oxidative stress but also suggestive of altered function of oxidatively modified proteins [28-30]. A representative western blot is shown in Figure 7F. Chronic ethanol

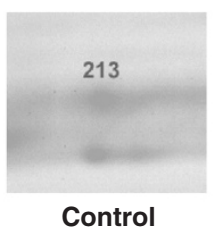

Proteome analysis

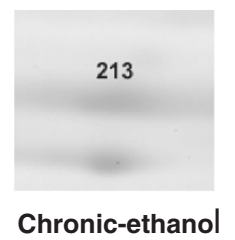

Chronic-ethanol

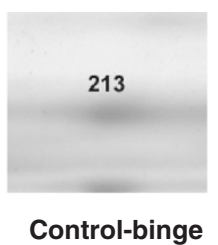

Control-binge

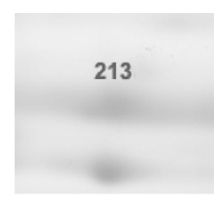

Chronic ethanolbinge
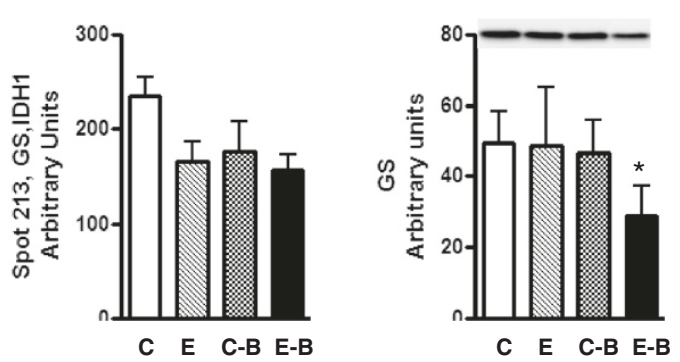

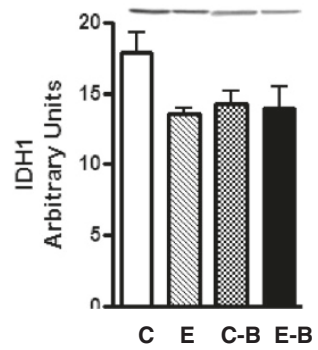

Western analysis

Figure 3 Quantitative analysis of cytosolic isocitrate dehydrogenase and glutamine synthetase from 2D gels and western immunoblot analysis. Rats were fed ethanol in liquid diet chronically for 4 weeks and then given 3 binge of ethanol and samples collected as in Figure 1. The levels of isocitrate dehydrogenase and glutamine synthetase were determined by western immunoblot. Control represents pair -fed animals given water for binge control. Values are mean $\pm S E(n=4$ rats). Western blot represents a typical experiment. *significant from control group $(p<0.05)$; C: Control (pair fed); E: Chronic ethanol; C-B: Control- ethanol binge; E-B: Chronic-ethanol binge. 

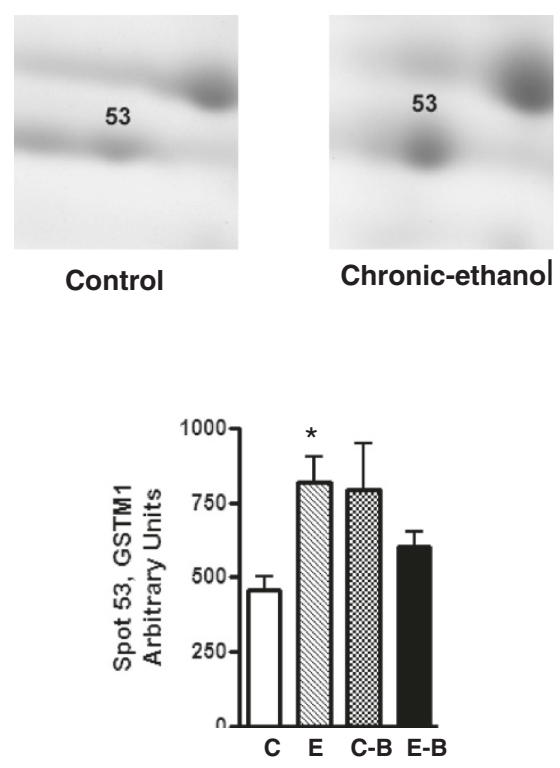

Proteome analysis
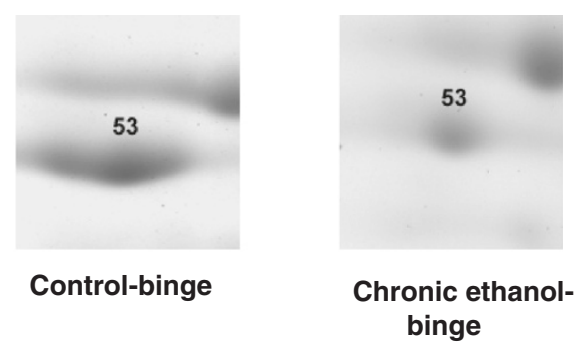

binge

Figure 4 Quantitative analysis of glutathione S-transferase M1 from 2D gels and western immunoblot analysis. Rats were fed ethanol in liquid diet chronically for 4 weeks and then given 3 binges before sample collection as detailed in Figure 1. The levels of glutathione transferase mu were determined by western immunoblot. Control represents pair -fed animals that were given water for binge control. Values are mean $\pm \mathrm{SE}$ $(n=3$ rats for each group). Western blot represents a typical experiment. * significant from control group ( $p<0.05)$; $\mathrm{C}: \mathrm{Control}(\mathrm{pair}$ fed); $\mathrm{E}$ : Chronic ethanol; C-B: Control- ethanol binge; E-B: Chronic-ethanol binge.

treatment alone caused increases in 4-HNE staining of low molecular weight whereas increases in 4-HNE staining of high molecular weight proteins were seen in the control binge group. In chronic ethanol-binge group there was an increase in 4-HNE staining of low molecular weight as well as of high molecular weight bands demonstrating increased oxidative stress in this group. Consistent with this pattern is the observation that CA3 protein levels decreased in the chronic ethanol group and further decrease in the chronic ethanol-binge (Figure 2). In the chronic ethanol, or control-binge groups a moderate increase in transaminase levels was noted. Its levels increased significantly in the ethanol- binge group suggesting augmentation of liver injury (Figure 7G). Recent studies have shown proapototic role for PDIA3 . This was supported by a decrease in PDIA3 expression and caspase 3 levels [31]. To examine this in our model we determined the expression of cleaved caspase 3 in different groups of ethanol treatment (Figure $7 \mathrm{H}$ ). Cleaved caspase 3 levels decreased in the chronic ethanol binge. We also examined the relevance of PDIA3. PDIA3 has been implicated in the modulation of histone H2AX phosphorylation. Decreased expression of PDIA3 by siRNA silencing resulted in decreased phosphorylation of H2AX [32]. Since H2AX phosphorylation has been implicated in DNA repair, we therefore examined histone $\mathrm{H} 2 \mathrm{AX}$ phosphorylation in the four treatment groups. Histone H2AX phosphorylation was significantly decreased in the chronic-ethanol binge group (Figure 7I).

\section{Discussion}

This is the first proteomic study of global alterations of proteins in liver from chronic ethanol-binge treated animals. The chronic ethanol binge model developed in this laboratory displays a number of features that are seen during human alcoholic liver injury (12). Therefore this model is useful for the investigation of the mechanism of binge induced enhancement of alcoholic liver injury. Using a proteomics approach, we have demonstrated changes in the levels of a number of liver proteins affected differentially by chronic ethanol, control-binge and chronic ethanol-binge. A notable aspect of this study is the identification of these protein targets in a single model. Previously these protein targets have been reported individually in separate studies. The fact that normal liver is resistant to acute ethanol binge [12] as seen in control-binge group in this study, we propose that the increased vulnerability to binge after chronic ethanol treatment and the accompanying alterations in proteins contribute to the amplification of injury.

We find a marked decrease in CA3 in chronic ethanol but not control-binge treated rats. Decrease in CA3 protein level after chronic ethanol administration has been reported [19], but the effects of acute ethanol binge or 


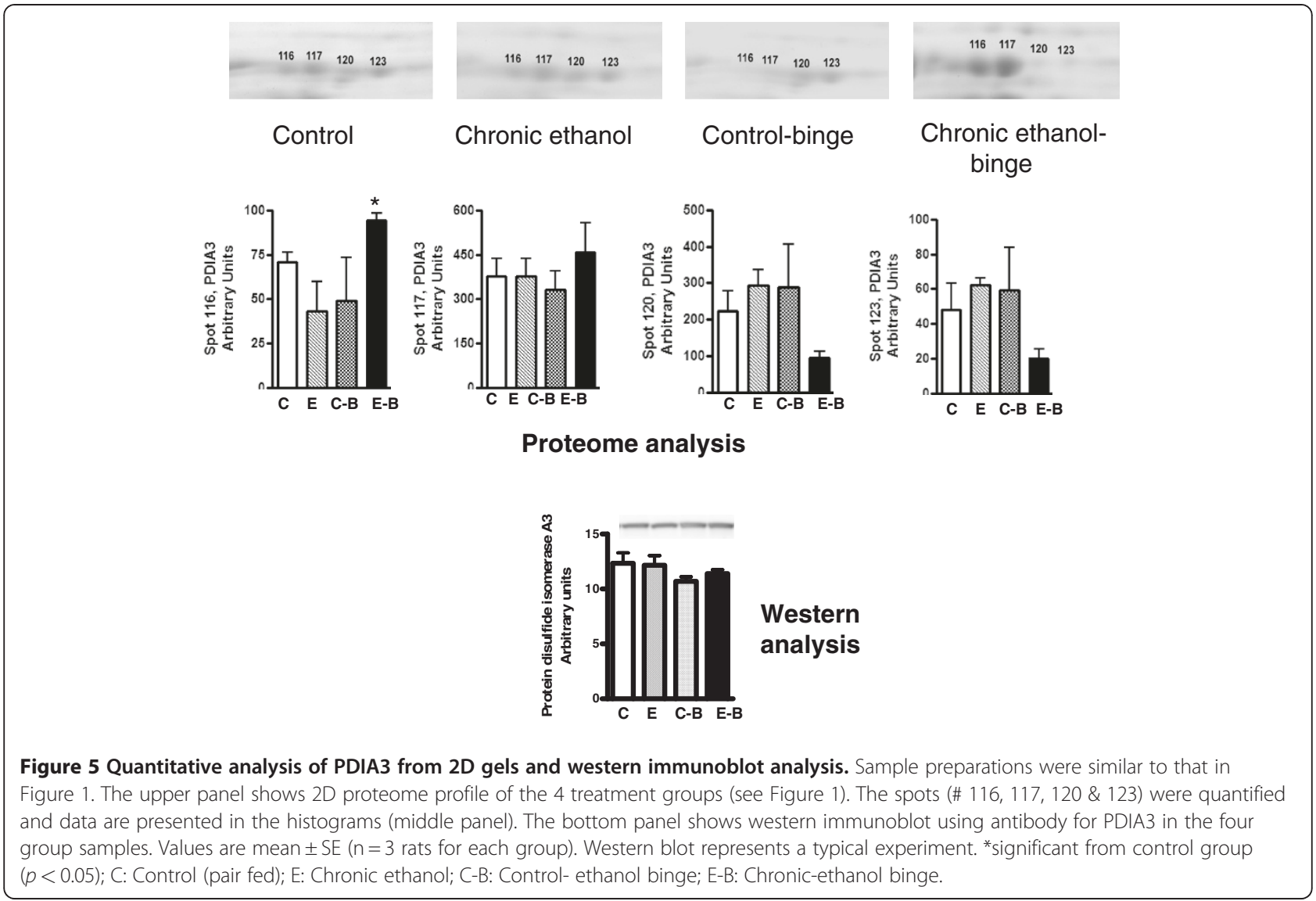

chronic-ethanol binge were not examined in that study. In another study, chronic ethanol treatment in rats decreased carbonic anhydrase mRNA levels [33] suggesting that the regulation of $\mathrm{CA} 3$ occurs at the level of transcription. CA3 is an enzyme that has negligible carbonic anhydrase activity compared to other isoforms of carbonic anhydrase [34]. It has been proposed to have a cytoprotective action against oxidative stress. In NIH/3T3 cells overexpressing CA3, steady state levels of reactive oxygen species are reduced and these cells were protected against hydrogen peroxide induced oxidative stress [35]. CA3 has also been shown to undergo reversible S-thiolation reactions with glutathione and reversible glutathiolation has been proposed to provide protection to the reactive cysteine residues in proteins from damaging oxidative reactions [36]. This assumes significance from studies supporting antioxidant role of CA3. A decrease in CA3 in the present study and augmentation of liver injury as demonstrated by increased transaminase favors the view that it has a protective role. Moreover, lipid peroxidation and oxidative stress has been shown to be increased in an experimental model of chronic ethanol followed by intraperitoneal administration of ethanol binge [37]. In the present study, we observed a marked decrease in CA3 levels in chronic ethanol-binge group that was accompanied by an increase in protein oxidation of low and high molecular weight proteins. There was a predominant increase of protein oxidation of low molecular weight in chronic ethanol group. Levels of oxidation of high molecular weight proteins increased in the controlbinge group. In this regard, it may be noted that carbonic anhydrase levels are far lower in humans and they are more susceptible to alcoholic liver injury than rats. Interestingly, female rats have far lower CA3 than male rats and female rats are more susceptible to alcoholic liver injury [38]. Increase in lipid peroxidation was accompanied by increased expression of CYP2E1 and ethanol induced CYP2E1 has been implicated in oxidative stress and steatosis [26,27]. However, the magnitude of increase in the CYP2E1 expression was dramatic in chronic-ethanol binge compared to chronic ethanol or binge alone. These findings suggest that chronic ethanol intake sensitizes liver for ethanol binge induced expression of CYP2E1 to a greater extent than direct effects of ethanol alone.

Isocitrate dehydrogenase 1 (IDH1) is a NADP-dependent enzyme that catalyzes the oxidative decarboxylation of isocitrate to $\alpha$-ketoglutarate $(\alpha-K G)$ in the cytoplasm/peroxisomes with the concomitant production of NADPH. It is proposed to play an important role in cellular defense against oxidative stress [39]. Treatment of hepatocytes 


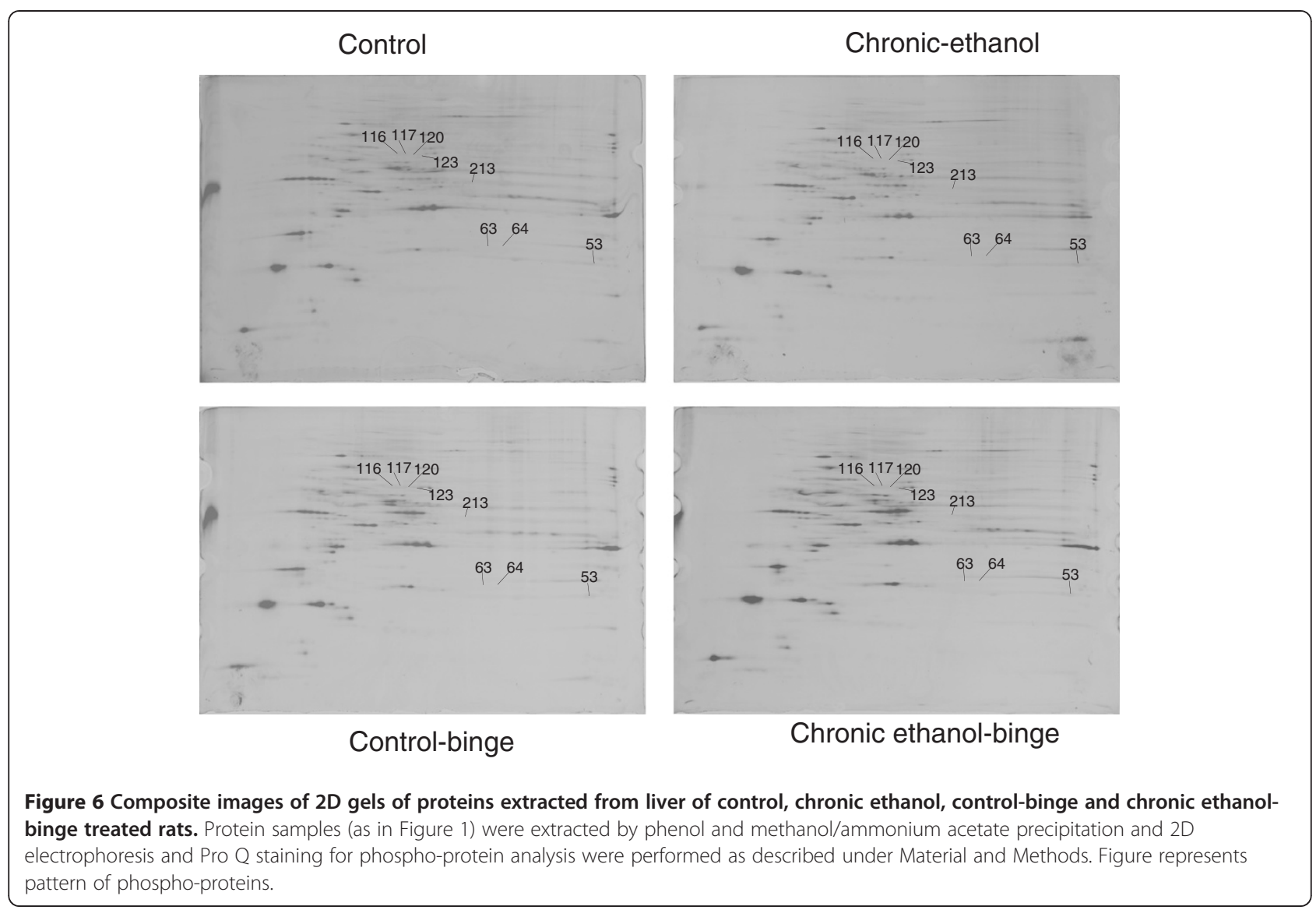

with ethanol or acetaldehyde decreased IDH1 activity, and knock down of IDH1resulted in potentiation of alcohol induced oxidative stress [40]. Decreased activity of IDH1 by oxidative stress has also been reported in liver [41]. In the present study, IDH1 was not significantly decreased after chronic ethanol intake or chronic ethanol-binge.

Glutamine synthetase catalyzes ATP-dependent amidation of glutamate to glutamine. It is responsible for the intrahepatic and inter tissue ammonia elimination/ detoxification occurring in the liver [42]. Glutamine synthetase has also been implicated in nucleotide synthesis and amino acid turnover [42]. Glutamine synthetase in normal liver is expressed by a small population of perivenous hepatocytes $[42,43]$. Decreased expression and activity of glutamine synthetase has been considered to be the sensitive indicator of liver injury and chronic ethanol intake has been shown to result in decreased glutamine synthetase activity [43,44]. Although, glutamine synthetase expression is modestly decreased after chronic ethanol, further decrease occurs after chronic-ethanol binge but not in control-binge. Decreased protein levels of glutamine synthetase in liver may contribute to increased plasma ammonia levels and exaggeration of central nervous system toxicity.
Glutathione S transferase (GST) is a superfamily of enzymes comprising several isoenzymes. The major GST subunits expressed in the adult liver are alpha (A1, A2 and A3) and Mu (M1 and M2). The Pi subunit of GST is only expressed in fetal hepatocytes, and during early stages of hepatocarcinogenesis [45]. GST Pi and M forms are drug metabolizing enzymes and play role in suppressing oxidative stress and reducing the levels of 4-hydroxynonenal [46]. Chronic ethanol exposure has been shown to cause both up- and down- regulation of GST M in vivo and in hepatocytes [46-49]. Accumulation of 4-hydroxynonenal after chronic ethanol treatment is considered to mediate liver injury via lipid peroxidation. Therefore, increased expression of GST that conjugates glutathione to a host of electrophiles [46] may represent an adaptive response induced by chronic ethanol. In this regard, GST M null phenotype has been shown to be associated with increased susceptibility to alcoholic liver injury [50]. In the present study, the expression of GST M1 was marginally increased after chronic ethanol or control ethanol binge, but the levels did not increase after chronic ethanol- binge suggesting compromised GST M1 after chronic ethanol-binge.

An intriguing finding in the present study is the change in the levels of different isoforms of PDIA3 in the 


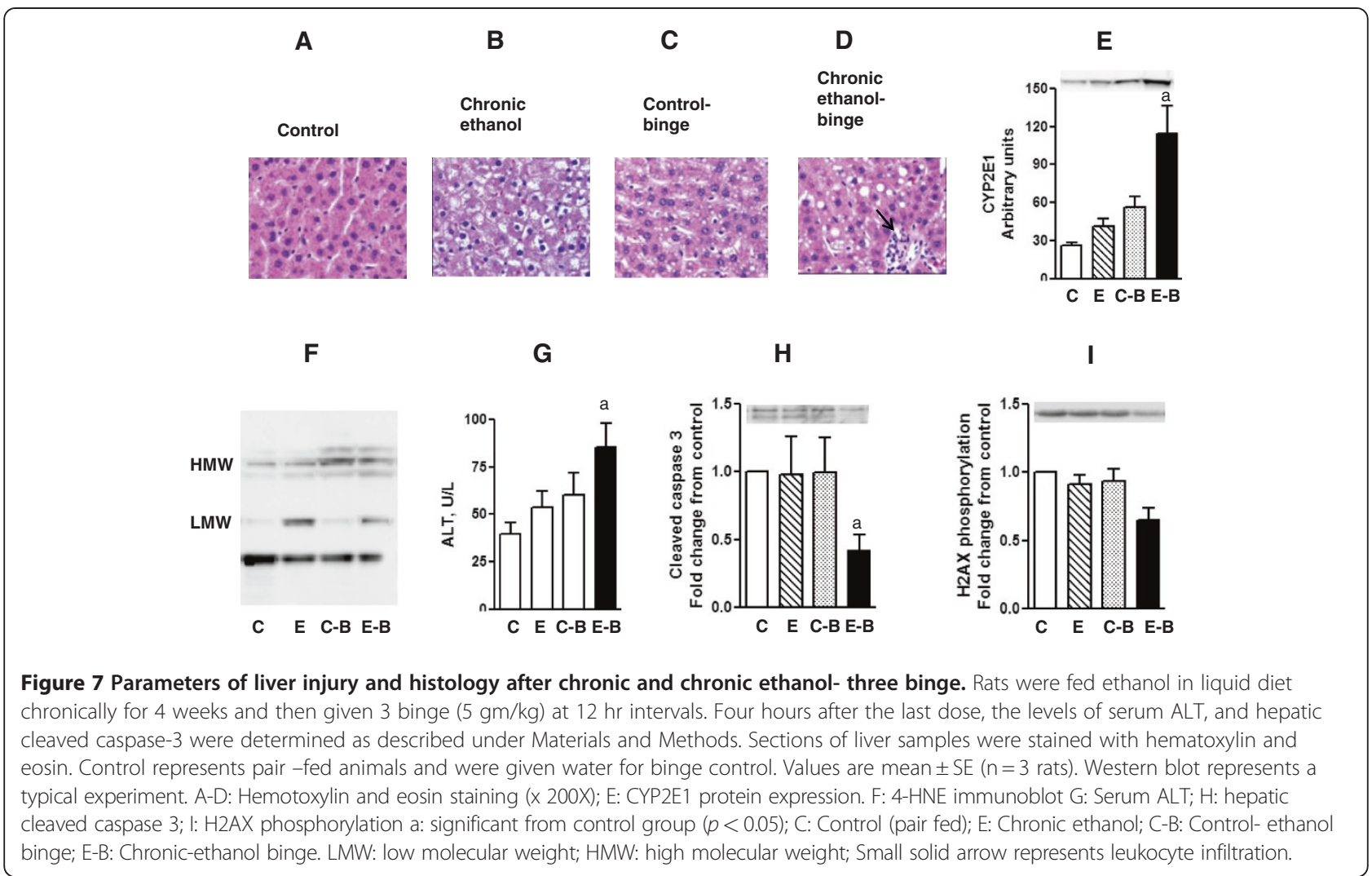

chronic ethanol-binge group compared to either chronic ethanol treated or control ethanol binge groups. PDIA3 belongs to the endoplasmic reticular chaperone protein family of protein disulfide isomerases [51]. These more negatively charged isoforms arise by either acetylation or phosphorylation of PDIA3. Recently, increased accumulation of phosphorylated forms of PDIA3 was shown after angiotensin II stimulation of vascular cells [52] or by prolonged fasting in rat liver [23]. Increased carbonylation of liver PDIA3 has been reported after chronic ethanol treatment in rats and this was accompanied by decreased function of the isomerase [53]. PDIA3 is emerging as an important regulator of cell function at multiple levels. It has been implicated in sorting of glycoproteins, quality control of endoplasmic reticular proteins, antigen processing by MHC proteins, control of STAT3 function, induction of apoptosis, and suppression of steatosis $[23,31,54,55]$. We have observed increased steatosis and suppressed apoptosis after chronic ethanol binge (Figure 7) with concomitant increases in the levels of PDIA3 acidic isoforms (Figure 5). Therefore, these results favor the role of PDIA3 in augmentation of liver injury by ethanol binge. Although suppression of apoptosis is considered as a survival mechanism, such phenomenon under the conditions of toxic insult such as ethanol may be accompanied by disruption of cell repair mechanisms such as repair of damaged DNA. DNA damage and impairment of DNA repair have been reported in animal and human alcoholic liver damage. Relevantly, knockdown of PDIA3 expression impaired phosphorylation of H2AX and DNA repair [32]. We have observed both decreased phosphorylation of H2AX and increase in the levels of acidic isoform of PDIA3 in chronic ethanol-binge group. It is also known that increase in acidic (phosphorylated) isoform of PDIA3 is related to decreased protein folding function of PDIA3 [23]. It is therefore plausible that in chronic ethanol-binge condition changes in PDIA3 disrupt protein folding, decrease H2AX phosphorylation resulting in a compromised DNA repair.

In summary, chronic ethanol and chronic ethanol binge display distinct signature profiles of protein expression. Differential expression of carbonic anhydrase 3, isocitrate dehydrogenase, glutathione S-transferase, glutamine synthetase, and protein disulfide isomerase protein 3 accompany increased susceptibility of chronic ethanol treated rat liver to binge induced injury. The consequences of these proteomic changes on the amplification of alcoholic liver injury by binge ethanol are schematically presented in Figure 8.

\section{Methods}

\section{Materials}

Male Sprague-Dawley rats (250-300 g) were purchased from Harlan Laboratories (Indianapolis, IN). ). The antibodies for carbonic anhydrase 3, protein disulfide 


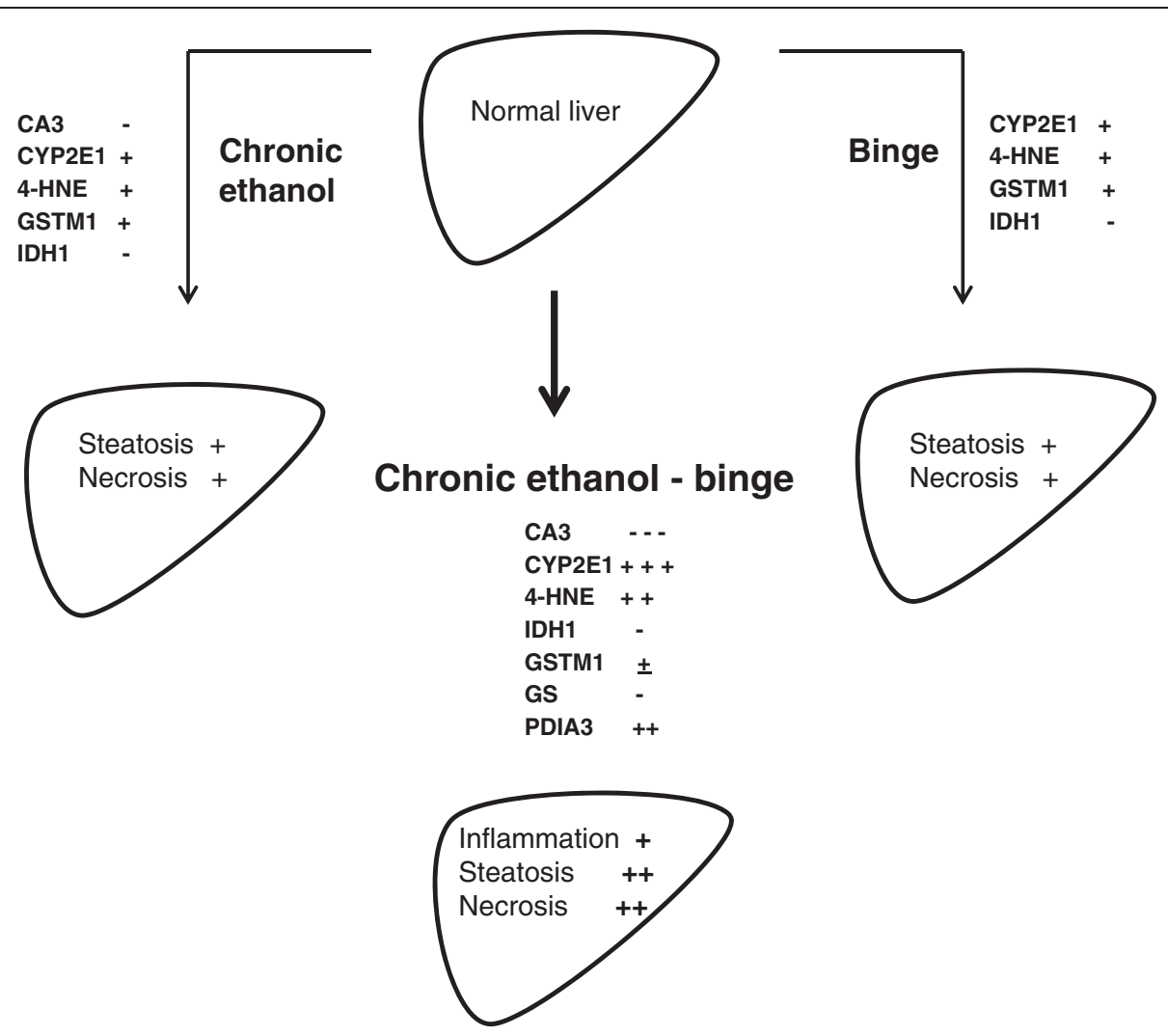

Figure 8 A flow diagram showing proteome changes and their relationshsip to liver injury by chronic ethanol binge in the rat model. This schematic figure depicts that binge, and chronic ethanol have their own effects, but chronic ethanol exposure renders liver more susceptible to amplification of injury by binge ethanol. The figure shows changes in the levels of CA3, CYP2E1, 4HNE, PDIA3 and other components after different treatments. Minus (-) and plus (+) symbols indicate the degree of decrease or increase, respectively.

isomerase associated protein 3, cytosolic isocitrate dehydrogenase, and glutamine synthetase were purchased from Santa Cruz Biotechnology Inc. (Santa Cruz, CA). Antibodies for histone $\mathrm{H} 2 \mathrm{AX}$ and cleaved caspase 3 were purchased form Cell Signaling (Beverly, MA). CYP2E1 antibody was from AbCam Cambridge, MA. Other reagents including protease inhibitor cocktail (Sigma p8340) and $\beta$-actin antibody were obtained from the Sigma-Aldrich (St. Louis, MO).

\section{Animal feeding for chronic ethanol-binge model of alcoholic liver injury}

Rats were housed under a 12-h/12-h light/dark cycle and were permitted ad libitum consumption of standard laboratory rat chow. After a one-week adaptation period, the animals were fed Lieber-DeCarli liquid diet (Dyets, Inc., Bethlehem, PA) [20]. Ethanol was progressively introduced into the liquid diet starting at $1.25 \%$ (wt./vol.) for day 1 , increased to $1.67 \%$ (wt./vol.) for day 2, to $2.5 \%$ (wt./vol.) for days 3 and 4 and, finally, maintained at 5\% (wt./vol.) for 4 weeks. Weight-matched littermates were pair-fed on the same liquid diet, except that the ethanol was replaced by dextrin/maltose (control) to maintain the isocaloric intake in the two groups. Each day, the previous day's ethanol diet intake was measured, and the pair-fed rats were fed same calorie of diet containing dextrin/maltose. After 4 weeks, rats were divided into four groups: control, chronic ethanol, control-binge , chronic-ethanol binge. Chronic ethanol-binge and control-binge groups had three doses (each $5 \mathrm{~g} / \mathrm{kg}$ body wt) of binge ethanol administered intragastrically every $12 \mathrm{hr}$. In the control group for chronic ethanol-binge, ethanol was replaced by water. Liver samples were collected $4 \mathrm{hr}$ after the last binge administration (Aroor et al, 2011), and immediately frozen in liquid nitrogen until further use. The animal care and protocol for their use were approved by the University of Missouri Animal Care \& Use Committee.

\section{Two dimensional electrophoresis}

Frozen liver samples were pulverized in liquid nitrogen using mortar and pestle. Tris-buffered phenol extraction solution was added to the powdered samples and proteins were extracted using phenol extraction protocol 
[56]. Protein pellets were resuspended in IEF buffer (8 M Urea, $2 \mathrm{M}$ thiourea, 4\% CHAPS, 2\% C7BzO, $100 \mathrm{mM}$ DTT, 2.2\% 2-HED). Solubilized samples were quantified using the EZQ protocol from Life Technologies (Grand Island, NY). One mg of protein sample was then applied to $24 \mathrm{~cm} \mathrm{pH} \mathrm{3-10} \mathrm{IPG} \mathrm{strips} \mathrm{for} \mathrm{passive} \mathrm{rehydration}$ overnight. The IPG strips were isoelectrically focused on the Protean IEF from Bio-Rad using the following parameters: $250 \mathrm{~V}$ for 250Vhr (rapid ramp), $1000 \mathrm{~V}$ for $500 \mathrm{Vhr}$ (rapid ramp), $8000 \mathrm{~V}$ for 2 hours (gradient), $8000 \mathrm{~V}$ for $80,000 \mathrm{Vhr}$ (rapid ramp), and then hold at $500 \mathrm{~V}$. The IPG strips were then placed on $12 \%$ SDSPAGE gels for the second dimension and ran using the Ettan Dalt 12 at $1 \mathrm{~W} /$ gel overnight. After the run, gels were stained with Pro-Q diamond stain and imaged using the Ettan DIGE imager. Gels were then post stained with Coomassie Brilliant Blue (G250) for the total protein and scanned using the UMAX powerlook flatbed scanner. Data were analyzed using Delta 2D system from Decodon after normalization. The identification of spots that showed distinct differences was based on aligning and matching of spots in gels and quantification of matched spots. The spots were also manually inspected to verify the accuracy of matching. We selected a protein spot that showed the least variation between the samples and this spot was used for normalization. All the protein spots chosen were present in all the gels. The results were also analyzed based on normalization to total spot volume and the pattern was similar to data analysis based on normalization to the protein spot with the least variation among the gels. For phosphoprotein analysis, the values were normalized for total protein.

\section{Analysis of proteins by mass spectrometry}

Spots were excised from the gel and subjected to trypsin digest using a standard in-gel digestion protocol [57]. The extracted peptides were lyophilized and resuspended in $3 \mu \mathrm{l}$ of $1 \%$ formic acid. A $0.5 \mu \mathrm{l}$ aliquot of the resulting peptides was mixed 1:1 with CHCA matrix $(5 \mathrm{mg} / \mathrm{mL}$ in $60 \%$ ACN, 0.3\% TFA, $10 \mathrm{mM}$ ammonium phosphate) and spotted onto the MALDI target. The instrument (Applied Biosystems 470 Proteomics Analyzer) was operated in positive ion mode and spectra were acquired over a mass range of 700 to $4000 \mathrm{Da}$. Peptide calibration standards (4700 calibration mix, Applied Biosystems) were used to calibrate the instrument in MS mode using six peptides of known mass. Calibration was achieved by the "plate model and default" mode for MS of six external calibrant spots. Additionally, internal (i.e. within the sample spot) calibration was achieved using trypsin autolysis peptides (where present). Note: although trypsin autolysis ions are useful for obtaining the best mass accuracy, these ions are automatically excluded from the database search conducted using the GPS Explorer.

Following an MS scan of each sample the 8 most abundant peptides were picked automatically for tandem MS (MSMS - peptide fragmentation) acquisition. Following this a database search was performed using V3.6 of GPS Explorer. Ions with $\mathrm{s} / \mathrm{n}$ ratios $>20$ (excluding trypsin autolysis) up to a max of 125 per spectrum were submitted for searches against mammal entries in the NCBInr database (last updated September $9^{\text {th }} 2010$ ) using the "combined MS + MSMS" function of the GPS Explorer software. The GPS Explorer software is integrated with the MASCOT V2.2 (www.matrixscience.com). Search parameters allowed: 1 trypsin miss-cleavage and the following modifications to peptides: carbamidomethyl cysteines, and methionine oxidation. MS mass tolerance was 100 ppm, MSMS mass tolerance was 0.2 Da.

Liquid Chromatography Tandem Mass Spectrometry analysis: Digests were diluted to $5 \mu \mathrm{l}$ with $1 \%$ formic acid and transferred to an autosampler vial and placed in the autosampler which was maintained at $4^{\circ} \mathrm{C}$. A portion of the sample $(3 \mu \mathrm{L})$ was loaded onto a $150 \mathrm{~mm} \mathrm{C18} \mathrm{CHIP}$ (Agilent Technologies cat\# G4240-62002). Peptides were separated and eluted from the analytical column with a continuous gradient of acetonitrile from 3 to $45 \%$ (in $0.1 \%$ formic acid) over 8 minutes. Following an MS scan of the eluting peptides, each cycle, the five most abundant peptides were subjected to peptide fragmentation (MSMS). Data across 17 minutes were collected. MSMS data were extracted and a peak list file (mgf - mascot generic format file) was generated using the qualitative analysis software. A search of NCBInr-limited to mammals was conducted using a local copy of MASCOT server V2.2. We selected only eight proteins by proteome analysis. The use of Coomassie blue staining and use of broader $\mathrm{pH}$ range might have resulted in the detection of lower number of spots. Our objective was to cover wide range of proteins and obtain adequate amount of protein for mass spectrometry analysis. Therefore, we have adopted this protocol. The limited number spot difference has also been reported for other proteome studies in chronic ethanol administration and endotoxin induced liver injury $[19,58]$.

\section{Statistical analysis}

Data are expressed as means \pm S.E.M. Statistical significance of the differences between means was assessed by Student's $t$ test. A probability of less than $5 \%$ was considered significant $(P<0.05)$.

\section{Abbreviations}

ALT: Alanine transaminase; CA3: Carbonic anhydrase 3; CBB: Coomassie brilliant blue; CYP2E1: Cytochrome P 450 2E1; GS: Glutamine synthetase; H2AX: Histone H2A X; HNE: 4-hydroxynonenal; GSTM1: Glutathione S- 
transferase, mu isoform; MS: Mass spectrometry; IDH1: Cytosolic isocitarate dehydrogenase; PDIA3: Protein disulfide isomerase associated protein 3.

\section{Competing interests}

The authors declare that they have no competing interests.

\section{Acknowledgements}

This work was supported in part by NIH grant AA11962 and AA16347.

\section{Author details}

'Department of Medical Pharmacology \& Physiology, University of Missouri, Columbia, MO 65212, USA. Proteomics Center, University of Missouri, Columbia, MO 65212, USA. ${ }^{3}$ Division of Biochemistry, University of Missouri, Columbia, MO 65212, USA.

\section{Authors' contributions}

ARA developed chronic ethanol binge animal model, conducted experiments, performed western immuno blots, did analysis of the results, and wrote the manuscript. LJR performed protein extraction, two dimensional electrophoresis and proteomic data handling. RJR conducted western blots, analysed the data and helped in manuscript writing. MBP did the MALDI-TOF-MS/MS analysis, contributed to the technical part on proteome experiments and supervised proteome experiments. SDS supervised the planning \& design of experiments, analysis \& interpretation of the results, and preparation of the manuscript. All authors read and approved the final manuscript.

Received: 2 December 2011 Accepted: 30 April 2012

Published: 30 April 2012

\section{References}

1. Adachi A, Brenner DA: Clinical syndromes of alcoholic liver disease. Dig Dis 2005, 23:255-263.

2. dela Hall MP, Lieber CS, DeCarli LM, French SW, Lindros $K O$, Jarvelainen $H$, Bode C, Parlesak A, Bode JC: Models of alcoholic liver disease in rodents. A critical evaluation. Alcoholism Clin Exp Res 2001, 25:254S-261S.

3. Kotesh A, Yang S, Lin H, Huang X, Diehl AM: Chronic ethanol exposure potentiates lipopolysaccharide liver injury despite inhibiting jun $\mathrm{N}$-terminal kinase and caspase 3 activation. J Biol Chem 2002, 44:13037-13044.

4. Schafer C, Parlesak A, Schutt C, Bode C, Bode JC: Concentrations of lipopolysaccharide binding protein, bactericidal/ permeability-increasing protein, soluble CD-14 and plasma lipids in relation to endotoxemia in patients with alcoholic liver disease. Alcohol Alcohol 2002, 37:81-86.

5. Fukui H: Relation of endotoxin, endotoxin binding proteins and macrophages to severe alcoholic injury and multiple organ failure. Alcoholism Clin Exp Res 2005, 29:172-179S.

6. Rivara PF, Jurkovich GJ, Gurney GJ, Seguin D, Fligner CL, Ries R, Raisvs VA Copass M: The magnitude of acute and chronic alcohol abuse in trauma patients. Arch Surg 1993, 128:907-913.

7. Crosse Kl, Anania FK: Alcoholic hepatitis. Curr Treat Options in Gastroenterol 2002, 5:417-423.

8. Ceccanti M, Attili A, Balducci B, Attilia F, Giacomelli S, Rotondo C, Sasso GF, Xerouchakis E, Attilia ML: Acute alcoholic hepatitis. J Clin Gastroenterol 2006, 40:833-841.

9. Zakhari S, Li TK: Determinants of alcohol use and abuse: impact of quantity and frequency patterns on liver disease. Hepatology 2007 46:2032-2039.

10. Mathurin $P$, Deltenre $P$ : Effect of binge drinking on the liver: an alarming public health issue? Gut 2009, 58:613-617.

11. Mathurin P, Beuzin PF, Louvet A, Carrie-Ganne N, Balian NA, Dalsoglio D, Trinchetet TC, Prevot S, Naveav S: Fibrosis progression occurs in a subgroup of heavy drinkers with typical histological features. Aliment Pharmacol Ther 2007, 25:1047-1054.

12. Aroor AR, Jackson DE, Shukla SD: Elevated activation of erk1 and erk2 accompany enhanced liver injury following alcohol binge in chronically ethanol-fed rats. Alcoholism Clin Exp Res 2011, 35:2128-2138.

13. Gstaiger M, Aebersold R: Applying mass spectrometry-based proteomics to genetics, genomics and network biology. Nat Rev Genet 2009, 10:617-627.

14. Mas RV, Fisher RA, Archer KJ, Maluf DG: Proteomics and liver fibrosis: identifying markers of fibrogenesis. Exper Trev Proteomics 2009, 6:421-431.
15. Uto H, Kanumra S, Takami Y, Tsubouchi H: Clinical proteomics for liver disease: a promising approach for discovery of novel biomarkers. Proteome Science. 2010, 8:70.

16. Beretta L: Liver proteomics applied to translational research in liver disease and cancer. Proteomics Clin Appl 2010, 4:359-361.

17. Bailey SM, Andringa KK, Landar A, Darley-Usmar VM: Proteomic approaches to identify and characterize alterations to the mitochondrial proteome in alcoholic liver disease. Methods Mol Biol 2008, 447:369-380.

18. Banerjee A, Russell WK, Jayaraman A, Ramaiah SK: Identification of proteins to predict the molecular basis for the observed gender susceptibility in a rat model of alcoholic steatohepatitis by $2-\mathrm{D}$ gel proteomics. Proteomics 2008, 8:4327-4337.

19. Kharbanda KK, Vigneswara V, McVicker BL, Newlaczyl AU, Bailey K, Tuma D, Ray DE, Carter WG: Proteomics reveal a concerted upregulation of methionine metabolic pathway enzymes, and downregulation of carbonic anhydrase-III, in betaine supplemented ethanol-fed rats. Biochem Biophys Res Commun 2009, 381:523-527.

20. Shepard BD, Tuma DJ, Tuma PL: Chronic ethanol consumption induces global hepatic protein hyperacetylation. Alcohol Clin Exp Res 2010, 34:280-291.

21. Andringa KK, King AL, Eccleston HB, Mantena SK, Landar A, Jhala NC, Dickinson DA, Squadrito GL, Bailey SM: Analysis of the liver mitochondrial proteome in response to ethanol and S-adenosylmethionine treatments: novel molecular targets of disease and hepatoprotection. Am J Physiol Gastrointest Liver Physiol 2010, 298:G732-G745.

22. Newton BW, Russel WK, Russel DH, Ramaiah S, Jayaraman: A liver proteome analysis in a rodent model of alcoholic steatosis. J Proteome Res 2009, 8:1663-1671.

23. Kita K, Okumura N, Takao T, Watanabe M, Matsubara T, Nishimura O, Nagai K: Evidence for phosphorylation of rat liver glucose-regulated protein 58, GRP58/ERp57/ER-60, induced by fasting and leptin. FEBS Lett 2005, 580:199-205.

24. Räisänen $S R$, Lehenkari $P$, Tasanen $M$, Rahkila $P$, Härkönen $P L$, Väänänen HK: Carbonic anhydrase III protects cells from hydrogen peroxide induced apoptosis. FASEB J 1999, 13:513-522.

25. Lee SM, Koh HJ, Park DC, Song BJ, Huh TL, Park JW: Cytosolic NADP (+)-dependent isocitrate dehydrogenase status modulates oxidative damage to cells. Free Radic Biol Med 2002, 32:1185-1196.

26. Cederbaum Al: Role of CYP2E1 in ethanol-induced oxidant stress, fatty liver and hepatotoxicity. Dig Dis 2010, 28:802-811.

27. Lu Y, Cederbaum Al: CYP2E1 and oxidative liver injury by alcohol. Free Radic Biol Med 2008, 44:723-738.

28. Smathers RL, Galligan JJ, Stewart BJ, Petersen DR: Overview of lipid peroxidation products and hepatic protein modification in alcoholic liver disease. Chem Biol Interact 2011, 192:107-112.

29. Sampey BP, Korourian S, Ronis MJ, Badger TM, Petersen DR: Immunohistochemical characterization of hepatic malondialdehyde and 4-hydroxynonenal modified proteins during early stages of ethanol-induced liver injury. Alcohol Clin Exp Res 2003, 27:1015-1122.

30. Poli G, Biasi F, Leonarduzzi G: 4-Hydroxynonenal-protein adducts: a reliable biomarker of lipid oxidation in liver diseases. Mol Aspects Med 2008, 29:67-71.

31. Xu D, Perez RE, Rezaiekhaligh MH, Bourdi M, Truog WE: Knockdown of ERp57 increases BiP/GRP 78 induction and protects against hyperoxia and tunicamycin-induced apoptosis. Am Physiol Lung Cell Mol Physiol 2009, 297:L44-L51.

32. Krynetskaia NF, Phadke MS, Jadhav SH, Krynetskiy EY: Chromatin-associated proteins HMG1/2 and PDIA3 trigger cellular response to chemotherapyinduced DNA damage. Mol Cancer Ther 2009, 8:864-872.

33. Tadic SD, ELM MS, Li H, Van London GJ, Subbotin VM, Whitcomb DC, Eagon PK: Sex differences in hepatic gene expression in a rat model of ethanolinduced liver injury. J Appl Physiol 2002, 93:1057-1068.

34. Mallis RJ, Hamann MJ, Zhao W, Zhang T, Hendrich S, Thomas JA: Irreversible thiol oxidation in carbonic anhydrase III: protection by S-glutathiolation and detection in aging rats. Biol Chem 2002, 383:649-662.

35. Zimmerman UJ, Wang P, Zhang X, Bogdanovich S, Forster R: Anti-oxidative response of carbonic anhydrase III in skeletal muscle. IUBMB Life 2004, 56:343-347

36. Lii CK, Chai YC, Zhao W, Thomas JA, Hendrich S: S-Thiolation and irreversible oxidation of sulfhydryls on carbonic anhydrase III during oxidative stress: a method for studying protein modification in intact cells and tissues. Arch Biochem Biophys 1994, 308:231-239. 
37. Kanbagli O, Balkan J, Aykac-Toker G, Uysal M: Hepatic Mitochondrial Prooxidant and Antioxidant Status in Ethanol-Induced Liver Injury in Rats. Biol Pharm Bull 2002, 25:1482-1484.

38. Parkkila S, Halsted CH, Villanueva JA, Vaananen HK, Niemela O: Expression of testerone dependent enzyme carbonic anhydrase III and oxidative stress in experimental alcoholic liver disease. Dig Dis Sci 1999, 44:2205-2213.

39. Brown SM, Upadhya R, Shoemaker JD, Lodge JK: Isocitrate dehydrogenase is important for nitrosative stress resistance in Cryptococcus neoformans, but oxidative stress resistance is not dependent on glucose-6-phosphate dehydrogenase. Eukaryot Cell 2010, 9:971-980.

40. Yang SE, Lee SM, Park WJ: Silencing of NADP-dependent isocitrate dehydrogenase gene enhances ethanol-induced cytotoxicty in Hep G2 cells. Arch Pharm Res 2010, 33:1065-1071.

41. Lee $J H$, Yang ES, Park JW: Inactivation of NADP + dependent isocitrate dehydrogenase by peorxynitrite. Implications for cytotoxicity and alcohol-induced liver injury. J Biol Chem 2003, 278:51360-51371.

42. Desjardins P, Rama Rao KV, Michalak A, Butterworth RF Rose C: Effect of portacaval anastomosis on glutamine synthetase protein and gene expression in Brain, liver and skeletal muscle. Metabol Brain Dis 1999 14:273-280.

43. Rouach H, Fataccioli V, Gentil M, French SW, Morimoto M, Nordmann R: Effect of chronic ethanol feeding on lipid peroxidation and protein oxidation in relation to liver pathology. Hepatology 1997, 25:351-355.

44. Abraham P, Wilfred G, Ramakrishna B: Oxidative damage to the hepatocellular proteins after chronic ethanol intake in the rat. Clin Chim Acta 2002, 325:117-125.

45. Khan AJ, Choudhuri G, Husain Q, Parmar D: Polymorphisim glutathione -Stransferases: a risk factor for alcoholic liver cirrhosis. Drug Alcohol Dep. 2009, 101:183-190.

46. Chen J, Schenker S, Henderson Gl: 4-hydroxynonenal detoxification by mitochondrial glutathione-S transferase is compromised by short-term ethanol consumption in rats. Alcoholism Clin Exp Res. 2002, 26:1252-1258.

47. Vanhaecke T, Lindros KO, Oinonen T, Coecke S, Debast G, Philips IR, Shepard EA, Vercruysse A, Rogiers $V$ : Effect of ethanol on the expression of hepatic glutathione $\mathrm{S}$ transferase. An in vivo/invitro study. Biochemical Phramacol 2000, 60:1491-1496.

48. Brind AM, Hurlstone A, Edrisinghe D, Gilmore I, Fisher N, Pirmohamed M, Fryer AA, Brind AM, Hurlstone A, Edrisinghe D, Gilmore I, Fisher N, Pirmohamed $M$, Fryer $A A$ : The role of polymorphisms of glutathione stransferase GSTM1, M3, Pi, T1 and A1 in susceptibility to alcoholic liver disease. Alcohol Alcohol 2004, 39:478-483.

49. Pal-Bhadra M, Bhadra U, Jackson DE, Mamatha L, Park PH, Shukla SD: Distinct methylation patterns in histone $\mathrm{H} 3$ at Lys-4 and Lys-9 correlate with up- \& down-regulation of genes by ethanol in hepatocytes. Life SCi 2007, 81:979-987.

50. Josephy PD: Genetic variations in human glutathione transferase enzymes: significance for pharmacology and toxicology. Human Genomics Proteomics 2010. ID 876940

51. Ellgaard L, Frickel EM: Calnexin, calreticulin, and ERp57: teammates in glycoprotein folding. Cell Biochem Biophys 2003, 39:223-247.

52. Ni M, Lee AS: ER chaperones in mammalian development and human diseases. FEBS Lett 2007, 581:3641-3651.

53. Panaretakis T, Joza N, Modjtahedi N, Tesniere A, Vitale I, Durchschlag M, Fimia GM, Kepp O, Piacentini M, Froehlich KU, van Endert P, Zitvogel L, Madeo F, Kroemer G: The co-translocation of ERp57 and calreticulin determines the immunogenicity of cell death. Cell Death Differ 2008, 15:1499-1509.

54. Tokutomi Y, Araki N, Kataoka K, Yamamoto E, Kim-Mitsuyama S: Oxidation of Prx2 and phosphorylation of GRP58 by angiotensin II in human coronary smooth muscle cells identified by 2D-DIGE analysis. Biochem Biophys Res Commun 2007, 364:822-830.

55. Turano C, Coppari S, Altieri F, Ferraro A: Proteins of the PDI family: unpredicted non-ER locations and functions. J Cell Physiol 2002, 193:154-163.

56. Mooney BP, Miernyk JA, Greenlief CM, Thelen JJ: Using quantitative proteomics of Arabidopsis roots and leaves to predict metabolic activity. Physiol Plant 2006, 128:237-250

57. Havlis J, Thomas H, Sebela M, Shevchenko A: Fast-response proteomics by accelerated in-gel digestion of proteins. Anal Chem 2003, 75:1300-1306.

58. Liu XW, Lu FG, Zhang GS, Wu XP, You Y, Ouyang CH, Yang DY: Proteomics to display tissue repair opposing injury response to LPS-induced liver injury. World J Gastroenterol 2004, 10:2701-2705. doi:10.1186/1477-5956-10-29

Cite this article as: Aroor et al:: A proteomic analysis of liver after ethanol binge in chronically ethanol treated rats. Proteome Science 2012 $10: 29$.

\section{Submit your next manuscript to BioMed Central and take full advantage of:}

- Convenient online submission

- Thorough peer review

- No space constraints or color figure charges

- Immediate publication on acceptance

- Inclusion in PubMed, CAS, Scopus and Google Scholar

- Research which is freely available for redistribution 